\title{
AI4R2R (AI for Rock to Revenue): A Review of the Applications of AI in Mineral Processing
}

\author{
Amit Kumar Mishra
}

check for

updates

Citation: Mishra, A.K. AI4R2R (AI for Rock to Revenue): A Review of the Applications of AI in Mineral Processing. Minerals 2021, 11, 1118. https://doi.org/10.3390/min11101118

Academic Editor: Lidia Auret and Kevin Brooks

Received: 8 August 2021

Accepted: 6 October 2021

Published: 12 October 2021

Publisher's Note: MDPI stays neutral with regard to jurisdictional claims in published maps and institutional affiliations.

Copyright: (c) 2021 by the authors. Licensee MDPI, Basel, Switzerland. This article is an open access article distributed under the terms and conditions of the Creative Commons Attribution (CC BY) license (https:/ / creativecommons.org/licenses/by/ $4.0 /)$.
Department of Electrical Engineering, University of Cape Town, Cape Town 7700, South Africa; akmishra@ieee.org

Abstract: In the last few years, jargon, such as machine learning (ML) and artificial intelligence (AI), have been ubiquitous in both popular science media as well as the academic literature. Many industries have tried the current suite of ML and AI algorithms with various degrees of success. Mineral processing, as an industry, is looking at AI for two reasons. First of all, as with other industries, it is pertinent to know if $\mathrm{AI}$ algorithms can be used to enhance productivity. The second reason is specific to the mining industry. Of late, the grade of ores is reducing, and the demand for ethical mining (with as little effect on ecology as possible) is increasing. Thus, mineral processing industries also want to explore the possible use of AI in solving these challenges. In this review paper, first, the challenges in mineral processing that can potentially be solved by AI are presented. Then, some of the most pertinent developments in the domain of ML and AI (applied in the domain of mineral processing) are discussed. Lastly, a top-level modus operandi is presented for a mineral processing industry that might want to explore the possibilities of using AI in its processes. Following are some of the new paradigms added by this review. This review presents a holistic view of the domain of mineral processing with an AI lens. It is also one of the first reviews in this domain to thoroughly discuss the use of AI in ethical, green, and sustainable mineral processing. The AI process proposed in this paper is a comprehensive one. To ensure the relevance to industry, the flow was made agile with the spiral system engineering flow. This is expected to drive rapid and agile investigation of the potential of applying ML and $\mathrm{AI}$ in different mineral processing industries.

Keywords: artificial intelligence; machine learning; mineral processing; sustainable mining; ethical mining; zero footprint

\section{Introduction: Pain-Points in Mineral Processing}

Mineral processing is the art of converting ores (rocks) in which the concentration of mineral might be low and boosting the concentration methodically (to be high enough to produce revenue).

Mineral processing usually follows four stages [1,2]

1. Ore preparation, which consists of steps, including selective mining (for various reasons), ore blending, and other pre-concentration techniques.

2. Comminution, where the size of the ore is reduced.

3. Sizing, where particles of certain sizes are passed on to the next stage.

4. Concentration, where particles with higher concentration are filtered out in stages so that the mineral concentration in the overall material goes up.

5. Dewatering, where the water from the particles is removed.

In addition to the above four conventional processes, mineral processing plants also need to take care of environmental issues. Ethical mining and metallurgy are witnessing active regulatory initiatives [3]. Taking care of the environmental implications of mineral processing is becoming an integral part of the operations [3]. Hence, Ethical Processing can be added as the fifth stage. 


\subsection{Some of the Current Challenges in Mineral Processing}

With the depletion of quality ores, mineral processing engineers have to deal with complex ores where either one or more of the following challenges exist $[4,5]$.

- There is a need to extract multiple minerals (complex composition) from the same ore. Or, there is a need to extract minerals of various concentrations from the same ore.

- The quality of the deposit is high but the size or accumulation is low.

- The quality of the deposit is low but the accumulation is high.

- Lastly, due to the recent interest in green and sustainable development, there is increasing importance on ethical and green mining with minimized climate and material footprint of the whole process chain [6]. This has also created a scarcity in ores.

The implication is often that the particle size needed for mineral liberation, at times, is as small as $1 \mu \mathrm{m}$. This makes mineral processing challenging and also increases the environmental footprint of mines.

How can ML and AI be of help? There are two implications of the current challenges in mineral processing.

1. No One Size Fits All: Traditionally, mineral processing techniques and machines have been uniform across operations. Even if there is fine-tuning involved for each site, the process parameters are not often changed. Ores were more or less consistent in their properties. When we are (figuratively) scraping the bottom of our deposit barrels, the properties of the ores are no longer consistent. This requires the real-time fine-tuning of process parameters. This is a domain where the application of ML and AI has shown considerable success in many domains [7-9]. This is expected to be one of the major applications of ML and $\mathrm{AI}$ in mineral processing shortly. These applications of AI would mostly not require major installation changes.

2. Reducing Human Error: Mineral processing plants are well-oiled processes. A small mistake can stop the process, and each hour costs an exorbitant amount of losses. Often, these mistakes are human-errors. There has always been a need to automate systems to avoid these. The need, now, is more acute. With the quality of ore diminishing, mineral processing plants are becoming more sophisticated. They come with numerous sensors whose values should be interpreted in real-time. These sensors are not human-perceivable. This means that these are not pictures or sounds for which humans have well-established sensory facilities. Interpreting data from these sensors requires a great deal of training and experience. This increases the chances of human error.

This is where ML and AI can really make a difference. Current generation deep learning (DL) algorithms are extremely good correlation extractors [10]. In terms of extracting correlations, they are better than humans [11,12], especially for nonoptical and non-audio types of sensory data. With a human in the loop (to make the executive decisions) and $\mathrm{AI}$ algorithms to interpret the sensory data, the chances of human-error can be reduced substantially. Sensors and AI are integral parts of most AI solutions. Hence, sensor and AI (SensAI) developments should always be done in a co-development model.

\subsection{Ever Expanding Meaning of Mineral Processing}

If we generalize the definition of mineral processing to encompass the processes needed to supply metals and materials to different applications, then we cannot ignore the following emerging branches of mineral processing.

- Mineral Processing for Urban Mining: Recycling and the circular economy (CE) have been taking an increasingly central position in the world order. Extracting metals and materials from urban waste can be called urban mining. The processing needed for this new kind of mining can also be called mineral processing. For example, harvesting Lithium from Lithium ion batteries, which is a prominent e-waste. Another interesting 
example is the use of living organisms to harvest metals from waste [13] (like the use of microorganisms to recover metal from discarded printed circuit boards [14]).

Recycling, especially in the case of certain metals, such as aluminium, is a well established business. The mineral processing needed for this traditional recycling is mostly simple. However, shortly, we will need to extract metals that are in very low concentrations from waste. This will require sophisticated mineral processing. AI has a major role to play in this. New companies, like Zen Robotics, are already pushing the boundaries in terms of sophisticated waste sorting using AI [15].

Secondly, in a circular economy, where there is no real beginning and end of materials, it will be difficult to exactly pinpoint the processes that can be called mineral processing. The preparation of ores for comminution is an integral part of mineral processing. In a circular economy, tracing the possible materials that will become a resource for the next slot of mining would also be a part of mineral processing. The role of SensAI is pivotal in tracing metals in circulation. For example, a group from Sweden has done made interesting innovations on the use of embedded sensors to trace metals in circulation [16].

- Renewable Mining: Renewables providing fuels and raw materials will become more common soon. If we can term the process of extracting materials and fuels from renewables as renewable mining then the preprocessing involved in these plants can also be termed as mineral processing. For example, work towards the use of biomass to produce polymeric materials, organic chemicals, and fuel $[17,18]$ has been going on since the 1970s. Bio-diesel [19] is one of the many materials, currently being produced from renewables.

The process chain of renewables (refer Figure 1 [20]) mostly involves steps that are the same as some of the steps followed in traditional mineral processing. SensAI will, again, play a pivotal role in these processes. AI can also be useful in recommending refined process chains for new kinds of renewable materials [16].

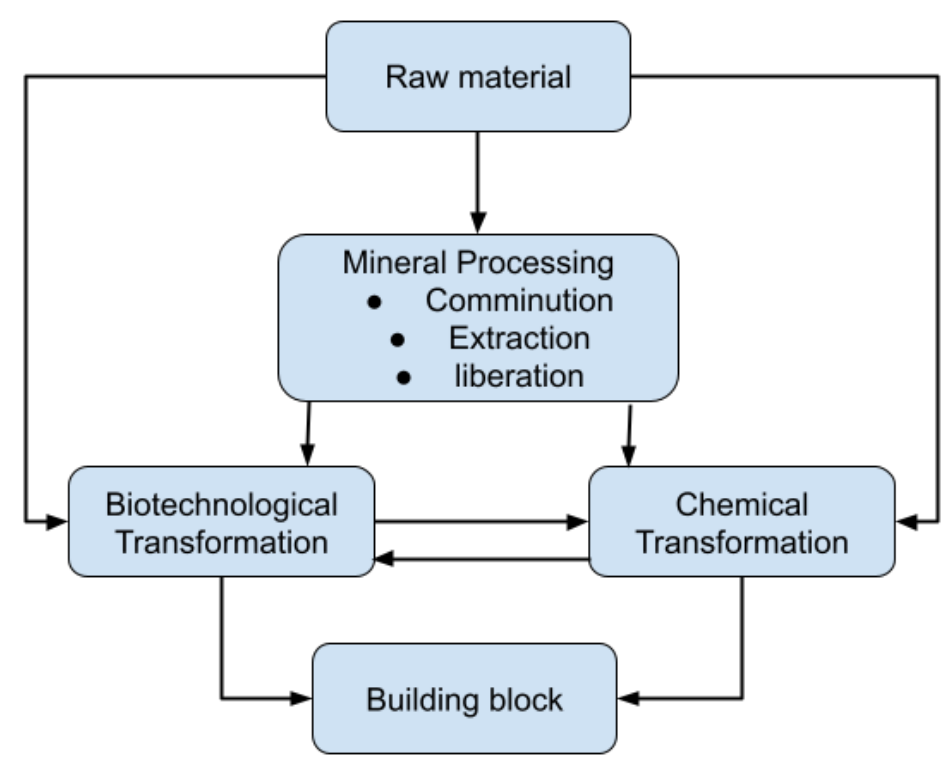

Figure 1. The typical process chain for renewables [20]. Many of the steps in this process chain are similar to the steps in conventional mineral processing.

The current review looks into the potential of machine learning (ML) and artificial intelligence (AI) in abetting the major challenges faced by mineral processing in all the above five stages. There are a few interesting reviews of a similar nature in the open literature $[21,22]$. The value proposition of the current review is both complementary and supplementary to the existing reviews. For example, Fu and Aldrich [21] gave a thorough review of the application of deep learning algorithms in mining. In this review, algorithms other 
than deep learning are also considered. In [22], McCoy and Auret presented a methodical review of the applications of ML in mineral processing.

The current review supplements their work with a discussion on the potential of AI in mineral processing. The applications of AI in alleviating the environmental impacts of mineral processing are also discussed. Lastly, the above reviews were written by domain experts working in the mineral processing industries. The author of this review is primarily an AI researcher. Hence, the presentation and flavour of the review will be different. In summary, it is recommended that readers refer to these reviews $([21,22]$ and the current review) together.

The rest of the paper is organized as follows. In the next section, some of the major approaches in ML and AI are discussed. In Section 3, some of the existing works that have explored the application of ML and AI algorithms in mineral processing are discussed. In Section 4, I discuss some of the futuristic applications of ML and AI in mineral processing (MP). Section 5 concludes the review. In this section, I also present a modus operandi for engineers and researchers who are planning to investigate the potential use of ML and AI in their work related to MP.

\section{A Review of AI and ML Algorithms}

To start, let us take a quick look at the machine learning (ML) and artificial intelligence (AI) algorithms and processes at our disposal. This is, by no means, a review of all the $\mathrm{ML}$ and AI algorithms and processes. The discussion is kept brief and pertinent to the application domain (viz. mineral processing).

Let us try to understand the meanings of three things (often used synonymously in popular literature), AI, ML, and Deep Learning (DL).

- Artificial Intelligence (AI): The wish to create something intelligent is as old as human intelligence itself. The endeavours were formalized by Turing in his elegant work [23] that gave us the famous Turing Test. I strongly suggest this work to anyone interested in AI. However, the Turing test is based on the concept of a universal Turing machine [24].

The meaning of "intelligence" itself is a hot field of research [25-27]. If and when intelligence can be defined analytically, implementing it using a Turing machine will be an easy follow-up step. Hence, some in the AI field are trying to approach the challenge from another angle. What if there is no mathematical framework to capture intelligence? The machine to implement AI need not be a Turing machine [28].

With this extremely short historical summary of the domain of AI, I note that AI is the super-set of all the pertinent endeavours. I, now, discuss ML and DL, which are subsets of AI.

- Machine Learning (ML): Machine learning, as a domain, involves algorithms that can use experiences and learn from them. Experiences, are mostly in the form of data that the ML algorithm uses to build models. These models are, in turn, used to perform a range of actions, such as pattern classification, recognition, anomaly detection, prediction, and regression. Arthur Samuel was the person who first used the phrase machine learning in his interesting work on modelling checkers [29].

- Deep Learning (DL): Deep learning is a subset of artificial neural networks (ANN), which is a subset of ML. ANNs have been around for more than seven decades. Hebbian networks [30] were one of the initial ANNs to be studied. Cybenko's work on the universal approximation capabilities of a sigmoid-based ANN [31] gave strong mathematical reason to expect a great deal from ANNs. However, there have been many brick walls that did not give traditional ANNs as many successes. With a few interesting innovations, deeper ANNs became more practical. One of the biggest advantages of deeper ANN was the fact that they can learn features on their own from data. Readers are suggested to refer to an excellent introduction to deep learning written by some of its founding fathers [10]. 
Soon, DL started to show amazing results in a range of domains. Due to the generous efforts from Facebook [32] and Google [33] in making their DL libraries free for all, the barrier to entry was reduced substantially as well. Lastly, DL has a strong brain-inspired narrative as well [34].

Now, let us discuss some of the major classes of ML algorithms. This shall, in no way, be a thorough review of ML algorithms. This will be a conceptual overview of some of the successful ML algorithms that might have the most potential in mineral processing. If the readers are interested in a good review of the domain of $\mathrm{AI}$ and $\mathrm{ML}$, they are recommended to some of the recent reviews, such as the Turin Lecture by Bengio, LeCun, and Hinton [35].

Machine learning algorithms can be grouped into two major categories based on their philosophy of development.

1. Generative $M L$ : This is the more traditional approach to ML. The underlying hypothesis is that measured data is always noisy. Hence, we cannot trust data! A model was needed that could describe the data and noise together. Traditionally, statistical models fit the requirements. Hence, in Generative ML, it is assumed that there is an underlying model that is generating the data. The more we understand this model, the more we know about the data. Statistical models are characterized by model parameters. Hence, statistical ML focuses on estimating these parameters and is also called Parametric ML. There are excellent textbooks on this subject [36,37].

The second revolution of generative ML came after the invention of the generative adversarial network (GAN) [38], which is a semi-parametric generative ML algorithm. The reason it is semi-parametric is that, even though it does not intend to build a statistical model, it still uses parametric statistics in comparing the generative and adversarial branches.

2. Discriminative $M L$ : In discriminative ML the data is trusted! The data is used to build ML models. The reason these algorithms are called discriminative is that in this the boundary that discriminates two classes or a class-limits is directly learned using the data.

Some of the oldest ML algorithms, like kNN [36], are discriminative in nature. Most ANN-based algorithms are discriminative in nature. One of the classic non-ANN discriminative ML algorithm is a support vector machine [37].

Some of the most successful discriminative ML algorithms include convolutional neural network (CNN) and recurrent neural network (RNN). The readers are referred to one of the classic reviews in DNN to learn more about these algorithms [10].

In addition to the above ML algorithms, I must also mention the Reinforcement Learning (RL) algorithms, which are one of the major contenders for AI systems that can learn from their own experience. These algorithms have shown excellent results in robotics [39] and also in some industrial control [40]. For industries, such as mineral processing where continuity of the process is of paramount importance, RL algorithms may not be very well suited. However, the trick is to find a sub-process in mineral processing that can be used as a process-in-loop for offline training of an RL model [41]. Offline RL algorithms hold a great deal of promise for industries, such as mineral processing.

\subsection{A Thumb-Rule to Choose AI/ML Algorithms}

New and exciting ML and AI algorithms are being proposed every month. To investigate the application of ML and AI solutions for a particular problem in mineral processing, one needs some algorithms to start with. From my experience, the following can be a safe chain of experiments.

1. The classic $k N N$ algorithm can always be taken as a starting point. It is easy to implement and can be used as a standard to compare other algorithms against.

2. Support Vector Machines (SVMs) are very powerful. Unlike most ANN-based algorithms, SVMs are analytically well-founded. In other words, they are not black-box solutions. Many times, when one does not have a sufficient amount of representative data for a problem, SVMs outperform DNNs. SVMs are also often faster than DNNs. 
In using SVMs, it is advisable to use as much domain knowledge as possible to extract useful features from the data. Feature engineering is not a forte of SVM.

3. In exploring DNNs, it is advisable to start with a CNN with a small number of convolutional units (CUs). This helps in analysing the filters learned by the CUs. This builds some amount of explainability into the solution.

4. If the problem at hand is time-based (e.g., predicting the output of a flotation cell) then recurrent networks, such as Long Short-term Memory (LSTM) networks, could be useful.

5. CNNs are very effective in feature learning. Hence, often, the architecture may have a CNN followed by an LSTM. A CNN followed by an SVM is also a potential solution.

6. If the amount of data is a major issue, then generative models can be used to augment data [42,43]. However, this is an area of active research. The design of a proper data augmentation system depends greatly on the domain knowledge.

\subsection{Future Tends}

In this subsection, I briefly discuss some of the potential innovations in the AI and ML research that will have large implications for mineral processing.

- eAI: AI algorithms need to be explainable, ethical, and empathetic. Explainable AI (xAI) is a major stream of development in the AI fraternity. One of the greatest developments in this direction has been the recent work toward bridging the gap between discriminative and generative AI. The development of generative AI models (GAN [38] being one of the most popular and powerful versions of it) started using neural networks not only to predict the discriminative boundaries of a given datapoint but also to generate the data itself. Thus far, this has been the forte of statistical machine learning. This, for me, is the starting point of XAI. The readers can refer to one of my informal articles [44] on interpretable AI for some further light discussion around xAI.

- Perception in the Loop: The importance of human perception is receiving a great deal of attention of late. Any real-life AI system will need to take this into account. I am not talking about making AI systems perceive things, such as humans [45]. That is a great goal for hard-AI scientists. I am talking about quantifying human perception and using it in training neural network models. Such perception-centric AI [46] systems will be crucial towards the adoption of $\mathrm{AI}$ in complex systems, such as industries and smart cities.

- Intuition-Based AI: The lack of a sufficient amount of data and the lack of generalized transferability are two challenges that I discussed above. Bio-inspired computational approaches have always given us new ways to look at problems. One brain-inspired approach that may enable AI algorithms to solve both the data and the transferability challenges is "intuition". Intuition-centered AI models [47] can use less amount of "right kind" of data to build robust models. It can also give directions to have an architecture that can leverage fusing symbolic and non-symbolic AI [48] to have a modus operandi to enable better transferability of models in the industrial ecosystem.

\section{Existing Research, Development \& Innovation (RDI) on the Use of ML and AI in Mineral Processing}

In this section, I discuss the recent trends in the use of $\mathrm{ML}$ and $\mathrm{AI}$ in mineral processing. It can be mentioned, as a disclaimer, that I am not able to discuss every work in the open literature in this section. However, it will be an endeavour to cover as many of the interesting applications as possible.

Ore preparation and geometallurgy is an integrated preceding step to mineral processing. In principle, an optimized geometallurgy process will optimize the mineral processing step. Physically, mineral processing often takes place in the mining site. Hence, ore-preparation and geometallurgy are integrated parts of mineral processing. Of late there has been much development in the use of ML and AI algorithms in ore preparation and 
geometallurgy. The reference [49] is a good review of the application of ML algorithms in ore preparation. The use of ML algorithms in ore-preparation has been an active field for more than a decade [50]. For further discussion on the application of ML and data science methods in geometallurgy can be found in [51,52].

\subsection{ML and AI in Comminution and Sizing}

Mineral distribution in ores is nonuniform. Hence, breaking them into smaller particles increases their concentration in some of the particles while reducing them in others. This process of comminution is often the first step in mineral processing. This is followed by the sizing step where particles are separated based on their sizes.

A common use of ML and AI in these two steps is in smart and automated control of the steps by monitoring of the size and mineral content in the particles [53-55].

This is a classic field where sensors and AI (SensAI) go closely together. The aim is to optimize the performance of the comminution step. For this, the sensor, usually, needs to sense either the size of the particles or the mineral signature of the particles both at the input and output of this step. Sensors with ML algorithms can be used to make sure that the size of rocks entering a mill and on the conveyor belt is not too big to cause failure [56]. ML-based monitoring of sizes [57-60] and quality (in terms of concentration of economic mineral) [61] of particles are some of the existing works in this domain.

The speed of execution or the computational complexity of these algorithms is crucially important as the decisions mostly need to be made real-time while the ores are moving on a conveyor belt. If the features are selected carefully then simple ML algorithms can also give very good results. For example, the researchers [57] designed two easy-to-compute features (viz. range and variance operators), which could be computed from the optical images of the ores. These, in turn, were used to give good classification performance in various classification exercises, such as separating different rock types to predicting the quality of the ore.

SensAI can also be used to monitor wear and tear in the comminution and sizing equipment $[62,63]$. As an extrapolation, the complete cycle of blasting, crushing, grinding and sizing can be optimized using ML tools $[64,65]$. This will be crucial shortly when the comminution unit might be required to operate underground as a mobile unit.

\subsection{ML and AI in Concentration and Dewatering}

In the concentration step, the overall percentage of minerals is enhanced further. This is usually done by using the physical and chemical properties of the minerals the particles. Common methods in this step include froth flotation, gravity concentration, and electromagnetic property-based separation. Like in comminution and sizing, the methodology involves the use of sensors (sometimes existing and sometimes new) to generate data about the process, which then is used by the ML algorithm to optimize the process and diagnose the process (for existing or possible (future) faults).

Froth flotation, being the most used method, has also witnessed several types of research in the application of ML algorithms. In froth flotation, the process parameters are, conventionally, controlled by human experts by looking at the texture of the froth. Automation of this step was difficult because of the poor performance of image-based ML algorithms in varying lighting conditions. Convolutional neural networks (CNN) have been shown to alleviate this pain point [66]. Of late, many other kinds of research have been published using ML algorithms in flotation monitoring and in the prediction (of yield quality) [67-69].

SensAI methods are also showing promising results in other areas of the concentration stage [70-72]. Dewatering is used to reduce the water content in the outputs from the concentration stage. ML-based algorithms are also showing potential in improving the efficiency of this stage $[73,74]$. 


\subsection{ML and AI in Operations}

Mineral processing is a complex process chain involving multiple systems in addition to the ones mentioned above. Automation and optimization of the overall operation and the sub-systems is a field where AI and ML algorithms play a major role. For example, in [75] the authors discuss an AI approach to automate the operation of dump trucks. References $[7,76]$ provide nice reviews of the use of ML and AI algorithms in the automation of various processes in the mining industry.

Like most industrial processes, the effective operation of a mineral processing plant is a complicated optimization task. The work described in [77] is a good overview of the integrated optimization needed to enhance the operations' parameters. Each step in a mineral-processing operation chain has multiple control parameters (operational indices) and multiple outputs (production indices). One of the most important production indices is the properties of the ore. For example, the size distribution of the ore at the end of each grinding step can be measured to control the grinders. The use of sensors, well-designed features, and ML/AI algorithms are methodologies increasingly used for this [78,79].

Clearly, the more often the properties of the ore in the process chain are measured, the more optimized the process is. However, using sensors at the output of each stage can sometimes be challenging. Hence, the better the process is modelled, the higher the possibility to use soft-sensor-based measurement to estimate the ore properties at each stage. The use of ML and AI algorithms have been promising in both modelling the stages [80-82] in mineral processing as well as the design of soft-sensors [83,84].

\subsection{ML and AI in Ethical and Green Mineral Processing}

Regulations around zero-footprint mining are under constant development. Mineral processes have various numbers of health, safety, and ecological implications. Mineral processing operations will be obliged by existing or upcoming regulations and standards to take care of the environmental and ecological footprints of the operations. Hence, these are becoming integral parts of mineral processing. The readers can refer to reviews, like [85,86], for a review of the application of ML and AI algorithms in the holistic mining domain with a focus on ethical mineral processing.

Some of these issues are listed below.

- Tailing: Tailing is a major harmful effect of mineral processing that can severely affect the safety of the local community, the local water resources, vegetation, and biodiversity. They can also affect the rainwater flow and hence affect the courses of the nearby rivers. A recent standard [87] by by the United Nations Environment Programme (UNEP), the Principles for Responsible Investment (PRI) and the International Council on Mining and Metals (ICMM) sets strict standards in terms of tailing management. This has resulted in the acceleration of research in this domain [88].

The use of specialized sensors, remote sensing and co-innovated AI models (SensAI) would be important parts of solutions to manage tailing [89,90].

In addition to mining industries, regulatory bodies will also be using SensAI solutions to monitor the effects of tailing of mines [91,92]. In this endeavour, remote sensing would play a major role [93,94].

- Wastewater Management: Most mineral processing operations are severely waterintensive. This affects the local water resources in two ways. It stresses the limited water resources. Wastewater from the plant can detrimentally affect the local reserves in detrimental way. Measurement is the key. The use of Internet of Things (IoT) based sensor-network as well as remote sensing would help in making sure that the both the water usage of the plant as well as quality (and quantity) of wastewater disposed of by the plant is strictly monitored.

It is encouraging to note that, of late, multiple earth-observing satellites have been launched with specialized sensors, such as multi-band synthetic aperture radar (SAR) $[95,96]$, soilmoisture mapping (SMAP) sensors [97] and hyperspectral sensors [98]. For mineral processing, hyperspectral sensors with AI hold tremendous possibilities [99]. 
- Hazardous Gas and Dust Emissions: Gases emitted by mineral processing can be dangerous when the concentration goes beyond a certain limit. For the safety of the workers and of the people living in the immediate vicinity, close monitoring of the levels of gas and dust would be highly advisable. Recent pieces of work have shown the potential of using SensAI for dust level monitoring [100-102]. Similarly, research in the domain of the use of SensAI for hazardous gas detection is growing rapidly [103-105]. The use of SensAI for gas and dust monitoring in a mineral processing plant is deemed to bear good results.

\section{The Futuristic Use-Cases}

Predicting the future is always a risky business. However, in a domain, such as ML and AI, where exciting algorithms are being invented almost every quarter and out-performing the previous ones, we must try. I briefly discuss a few potential new uses of ML and AI in mineral processing industries.

\subsection{Chemical Discovery}

The discovery of new compounds with interesting properties has been an active field of research in health-science. There are two reasons for this. The time and effort taken in testing each medical compound are huge. Secondly, the range of different properties that are exhibited by organic compounds slightly different in structure from each other is often numerous. Drug discovery or drug design using machine learning has been active for more than two decades [106-108]!

As the ores become more complex, the chemical additives required in each stage of mineral processing need to be more matched to the exact contents of the ore. This is a rich potential field for innovation using AI-enabled chemical discovery. In addition to this, if we expand the context of mineral processing to renewables as well, then that will require the use of organic compounds for which AI has already been proved to be efficient in designing the right catalysts.

\subsection{Process Diagnosis, Recommendation and Modification}

Most of the steps in mineral processing are difficult-to-model physiochemical processes. For example, froth flotation, used often in many mineral processing plants, is a very complicated process. Researchers have spent decades in trying to model it as well as possible [109] so that the right process-parameter fine-tuning can be applied to increase the efficiency. Due to the range of physical and chemical interactions in frothing, the mathematical models can often be complicated and still heuristic.

Can AI be used to bolster the modelling efficiency of mineral processing steps? Simulation and modelling based on machine learning has already proved effective in many domains [110]. There are two major types of process-modelling endeavours using machine learning.

1. End to End Modelling: In this approach, the complete process is modelled as a blackbox. Depending on how well sampled the data-space is (depending on strategically placed sensors and sensing-timing), deep learning networks can learn a process quite well. Especially in industrial processes where the chances of abrupt changes are lower, deep learning can be a powerful solution.

2. Latent Parameter Modelling: In this approach, the machine learning algorithm endeavours to model not only the end-to-end characteristics of a process but also its latent causes. The Bayesian mixture model has been particularly successful in modelling latent factors (which may or may not have phenomenological interpretation) [111,112]. The second set of algorithms capable of modelling latent factors are the encoderdecoder family of DL algorithms. Due to their ability to extract latent factors, they are used more often in process-modelling [113,114].

To end this subsection, an interesting success story of deep learning (DL)-based ML can be mentioned. The use-case is the domain of speech recognition. Speech recognition is one of the oldest and classic domains of machine learning. Over the decades, this domain 
experienced a great deal of meticulous and methodical development. In terms of modelling, conventional speech processing has been very close to physiology in its approach [115-117] However, since 2012 (when Google demonstrated the performance of deep learning in large vocabulary speech recognition (LVSR) [118]), most speech recognition systems have slowly started using deep learning instead of the conventional statistical approaches. This does not mean that the domain knowledge gathered over decades was useless. That knowledge helped immensely in designing these DL models.

ML and AI models can, potentially, complement and supplement existing process models in mineral processing. As these models are quicker to develop and easier to refine, they can prove valuable in the ever-changing landscape of mineral processing. Along with the interesting algorithms mentioned above, researchers can also work on the designing of a novel sensor suite, and together the SensAI system can be used to create digital twins of the process plants $[119,120]$.

Lastly, such models can be used not only to control a process. They can be used to diagnose faults, suggest modifications, and also to recommend newer (potentially more efficient) designs.

\section{Suggested Modus Operandi to Investigate AI for a Specific Mineral Processing Challenge}

In the current section, I discuss some of the steps that are highly recommended when working on a new project around the use of $\mathrm{AI}$ in mineral processing. Most of these steps hold true for other industries (in addition to mineral processing industries) as well. Secondly, every new application is different in its own right. Technology is maturing at a rapid pace. The time constant for the knowledge doubling curve [121] is becoming shorter.

Hence, the reader is recommended to fine-tune these recommendations if needed. Lastly, AI is not a panacea. It can alleviate many challenges, but not all. The more well defined our challenge is and the more domain knowledge brought into the project, the better and more reliable the performance of the solution will be. Figure 2 shows the steps in a flow-chart representation.

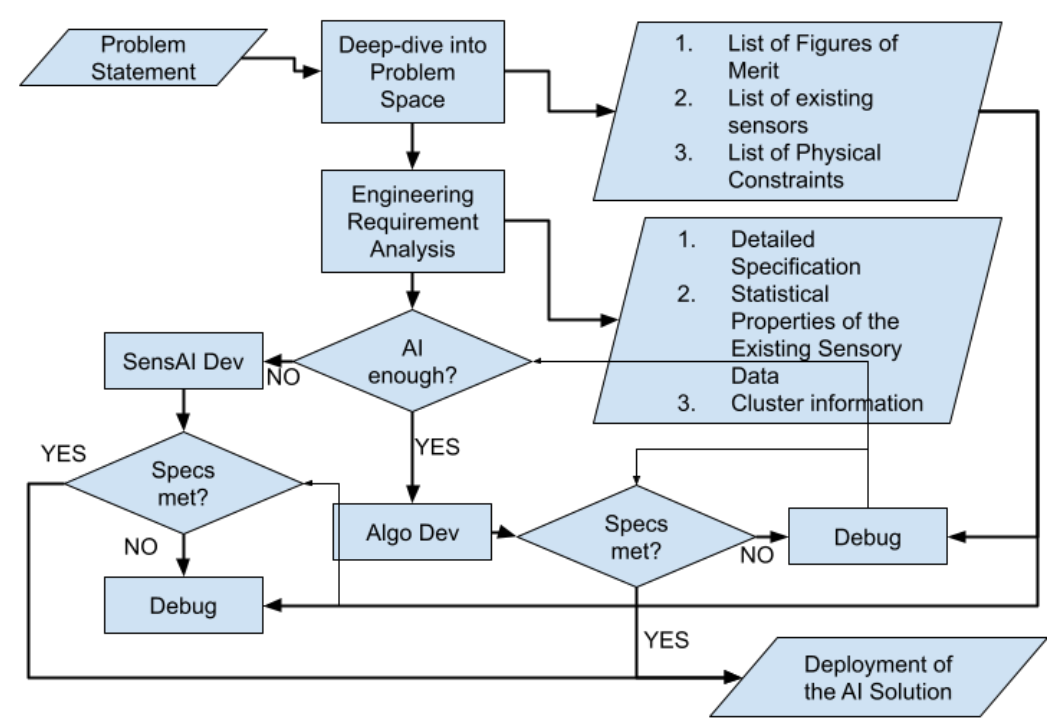

Figure 2. The suggested process to implement AI-based solutions for a mineral processing-related challenge. The modus operandi follows an agile approach and a spiral design flow. This is why there are multiple loops in the flow.

1. Immersion in the Problem Space (data): Design thinking [122] methodologies strongly suggest one to spend as much time as one can in the problem space. This is of particular importance in industrial AI innovation projects. The following are some of the important steps one can take in this phase. 
Figures of Merit: The famous theorem of "no free lunch" [123,124] roughly tells us that there is no universal algorithm that can solve all the problems. An algorithm can be good at a certain task but will not perform as well in other problems. The corollary of this is that, to find an algorithm that performs very well, the problem needs to be defined very well.

Defining the problem, invariably, means finding the best set of figures of merits. A classic example is target detection algorithm design in radar systems [125]. For a radar systems, the probability of a false alarm is more important than the probability of detection.

Given an AI-challenge in mineral processing, we need, first of all, to list how the decision of the proposed AI system will affect the operations.

In $\mathrm{ML}$, it is relatively easy to take care of a set of figures of merit each of which has different importance. One of the ways to achieve this is by using a weighted sum of loss function [126-129].

The Data Space: Exploratory data analysis $[130,131]$ is always recommended as a step to spend some time in. Irrespective of the problem at hand, some data can always be obtained from the process plant. Analysis of the statistics of the data and the clustering of the data from different sources are some of the recommended steps. First of all, most ML algorithms work the best with Gaussian data. Hence, it is always useful to know if the data distribution is going to deviate substantially from a normal distribution [132]. Secondly, even using simple principal component analysis (PCA) [133] to visualize the data in three dimensions can lead to interesting data models [134].

At this stage, it is also advisable to endeavour to find out as much as possible about pertinent statistical bounds [135], viz. Cramér-Rao bound (CRB) [136], Fisher Information [137] etc.

The Physical Space: While investigating the feasibility of AI for any industrial application it is highly recommended to visit the physical space. The more we know about the actual physical space and operation, the better equipped we will be to take care of interesting bugs that may arise later. An infamous case study is the mysterious signal detected by radio astronomers in Australia, which was later identified as radio frequency interference coming from an old microwave oven [138]!

The end of this phase should also give a list of detailed specifications that have to be met by the AI-solution.

2. AI or SensAI?: After having a deep dive into the problem space, one needs to decide on the next important choice. AI systems can be implemented either using existing sensory data or by using new sensors. The first case where we already have the sensors in place will involve innovations in the algorithm space only and can be called AI-only development. In the second case where the most informative data is not available, we can go for the installation of new sensors. In this case, the sensors and AI algorithms are co-innovated and co-developed. For example, hyperspectral imaging [139] and high-energy ultrasound sensors [140] have shown good potential in ore quality analysis. Combined with ML, hyperspectral imaging can be used in many stages of mineral processing to customize the process parameters depending on the ore quality [141].

However, in most heavy industries, any change to the existing setup is extremely costly (especially as it can lead to down time). Hence, the engineers are highly encouraged to thoroughly investigate what can be achieved by using the existing sensory-data. The use of AI can also, in some cases, aid us in generating secondary data that correlates well to a non-existing sensor using data from existing sensors. This is sometimes called soft-sensors [142].

At other times, it might be possible to design a SensAI system that uses data from low-fidelity sensors to give direct-decisions with high-fidelity. This paradigm can be called application-specific instrumentation (ASIN) [143,144]. 
3. Development, Debug and Deployment: This last step is not common to any engineering development. Depending on the route chosen in the previous step the development would either be algorithm development or a co-development of a SensAI system. If the performance of the solution meets the specifications, then it can be taken to commissioning. However, this rarely happens in the first iteration. In the debug process, the insights gathered in the deep-dive phase can be used effectively. At times, there might be statistical limits preventing us from achieving the specifications. In that case, we would need to change the sensors or the setup or need to adjust the specifications.

\section{Conclusions}

Mineral processing is experiencing exciting research initiatives to answer the everincreasing expectations from users and policymakers and the ever-decreasing quality of ores. In addition, we are also experiencing exciting times when newer and stricter regulations and policies are being enacted to encourage and enforce ethical mining and reduced material footprint. At the same time, the new wave of circular economy is pushing the boundaries of the conventional definition of ores and mineral processing.

Machine learning and AI-based solutions will be an integral part of innovations to progress the domain of mineral processing in the milieu described above. This review paper strives to present a modern review of the applications of $\mathrm{ML}$ and $\mathrm{AI}$ in the ever-changing landscape of mineral processing.

The landscape of ML and $\mathrm{AI}$ is overwhelming due to the accelerating growth in the amount of research happening recently. Section 2 of this paper strives to alleviate this by giving a holistic review of ML and AI algorithms without using heavy mathematics. This review should be a good starting point for any engineer planning to start working in an applied ML project. The review leaves the reader with a decent number of good references in case they want to dive deeper.

The domain of mineral processing is broad. There are a number of good investigations happening trying to apply ML and $\mathrm{AI}$ in all the sub-domains of mineral processing. Consolidating them all was a challenge. Section 3 endeavours to do this by grouping some of the major recent developments involving the application of $\mathrm{ML}$ and $\mathrm{AI}$ in the different sub-processes of mineral processing. ML and AI are areas of intense research initiatives and an accelerating number of solutions coming out every quarter. Hence, instead of discussing particular algorithms, the focus was on the discussion of the trends and methodologies.

As mentioned above, the milieu of mineral processing is changing. This is creating a range of futuristic challenges. Section 4 discusses the potential use of ML and AI in solving some of these futuristic challenges in mineral processing.

Lastly, in Section 5, an agile and spiral modus operandi is presented consolidating the application of ML and AI in any challenges in the mineral processing industry. To keep it pertinent to both industry and academic researchers, the process covers the full flow of investigation starting from the initial pre-study to data investigation to suggestions to smoothen the deployment of an ML or AI solution.

Funding: This research received no external funding.

Conflicts of Interest: The author declare no conflict of interest.

$\begin{array}{ll}\text { Nomenclature } \\ \text { AI } & \text { Artificial Intelligence } \\ \text { ML } & \text { Machine Learning } \\ \text { CE } & \text { Circular Economy } \\ \text { SensAI } & \text { Sensing and AI } \\ \text { DL } & \text { Deep Learning } \\ \text { ANN } & \text { Artificial Neural Network }\end{array}$




$\begin{array}{ll}\text { CNN } & \text { Convolutional Neural Network } \\ \text { GAN } & \text { Generative Adversarial Network } \\ \text { RNN } & \text { Recursive Neural Network } \\ \text { SVM } & \text { Support Vector Machine } \\ \text { CU } & \text { Convolutional Unit } \\ \text { LSTM } & \text { Long Short-Term Memory } \\ \text { RDI } & \text { Research, Development and Innovation } \\ \text { UNEP } & \text { United Nations Environment Programme } \\ \text { ICMM } & \text { International Council on Mining and Metals } \\ \text { PRI } & \text { Principles for Responsible Investment } \\ \text { SAR } & \text { Synthetic Aperture Radar } \\ \text { SMAP } & \text { Soil-Moisture Mapping } \\ \text { LVSR } & \text { Large Vocabulary Speech Recognition } \\ \text { PCA } & \text { Principal Component Analysis } \\ \text { CRB } & \text { Cramèr-Rao bound } \\ \text { ASIN } & \text { Application-Specific Instrumentation } \\ \text { IoT } & \text { Internet of Things }\end{array}$

\section{References}

1. Kelly, E.G.; Spottiswood, D.J. Introduction to Mineral Processing; John Wiley \& Sons Inc.: Hoboken, NJ, USA, 1982.

2. Gupta, A.; Yan, D.S. Mineral Processing Design and Operations: An Introduction; Elsevier: Amsterdam, The Netherlands, 2016.

3. Mudd, G.M. Sustainable/responsible mining and ethical issues related to the Sustainable Development Goals. Geol. Soc. Lond. Spec. Publ. 2021, 508, 187-199. [CrossRef]

4. Trends and Challenges for Technology in Mineral Processing. Available online: https://www.australianmining.com.au/news/ trends-challenges-technology-mineral-processing/ (accessed on 17 September 2021).

5. Evolving to Meet Future Challenges in Mining and Minerals Processing. Available online: https://www.hatch.com/About-Us/ Publications/Blogs/2020/02/Evolving-to-meet-future-challenges-in-mining-and-minerals-processing (accessed on 17 September 2021).

6. Jiskani, I.M.; Cai, Q.; Zhou, W.; Shah, S.A.A. Green and climate-smart mining: A framework to analyze open-pit mines for cleaner mineral production. Resour. Policy 2021, 71, 102007. [CrossRef]

7. Ali, D.; Frimpong, S. Artificial intelligence, machine learning and process automation: Existing knowledge frontier and way forward for mining sector. Artif. Intell. Rev. 2020, 53, 6025-6042. [CrossRef]

8. Min, Q.; Lu, Y.; Liu, Z.; Su, C.; Wang, B. Machine learning based digital twin framework for production optimization in petrochemical industry. Int. J. Inf. Manag. 2019, 49, 502-519. [CrossRef]

9. Kwame Osei, E. Machine Learning-Based Quality Prediction in the Froth Flotation Process of Mining. Master's Thesis, Dalarna University, Falun, Sweden, 2019.

10. Hinton, G.; LeCun, Y.; Bengio, Y. Deep learning. Nature 2015, 521, 436-444.

11. Froomkin, A.M.; Kerr, I.; Pineau, J. When AIs outperform doctors: Confronting the challenges of a tort-induced over-reliance on machine learning. Ariz. Law Rev. 2019, 61, 33.

12. Sejnowski, T.J. The unreasonable effectiveness of deep learning in artificial intelligence. Proc. Natl. Acad. Sci. USA 2020, 117, 30033-30038. [CrossRef]

13. Staicu, L.C.; Stolz, J.F. Microbes vs. metals: Harvest and recycle. FEMS Microbiol. Ecol. 2021, 97, fiab056. [CrossRef]

14. Argumedo-Delira, R.; Díaz-Martínez, M.E.; Gómez-Martínez, M.J. Microorganisms and plants in the recovery of metals from the printed circuit boards of computers and cell phones: A mini review. Metals 2020, 10, 1120. [CrossRef]

15. Lukka, T.J.; Tossavainen, T.; Kujala, J.V.; Raiko, T. Zenrobotics recycler-robotic sorting using machine learning. In Proceedings of the International Conference on Sensor-Based Sorting (SBS), Aachen, Germany, 11-13 March 2014; pp. 1-8.

16. Carlsson, R.; Elmquist, L.; Johansson, C. Cast metal with intelligence-from passive to intelligent cast components. In Proceedings of the VIII ECCOMAS Thematic Conference on Smart Structures and Materials (SMART), Madrid, Spain, 5-8 June 2017.

17. Sarkanen, K.V. Renewable resources for the production of fuels and chemicals. Science 1976, 191, 773-776. [CrossRef]

18. Narayan, R. Biomass (renewable) resources for production of materials, chemicals, and fuels: A paradigm shift. In Emerging Technologies for Materials and Chemicals from Biomass; American Chemical Society: Washington, DC, USA, 1992.

19. Amin, A. Review of diesel production from renewable resources: Catalysis, process kinetics and technologies. Ain Shams Eng. J. 2019, 10, 821-839. [CrossRef]

20. Peuker, U.; Kwade, A.; Teipel, U.; Mütze, T. Mineral Processing Mineral, Renewable and Secondary Raw Material Processing-Current Engineering Challenges; ProcessNet Subject Divisions: Frankfurt, Germany, 2012.

21. Fu, Y.; Aldrich, C. Deep Learning in Mining and Mineral Processing Operations: A Review. IFAC-PapersOnLine 2020, 53, 11920-11925. [CrossRef]

22. McCoy, J.T.; Auret, L. Machine learning applications in minerals processing: A review. Miner. Eng. 2019, 132, 95-109. [CrossRef]

23. Turing, A.M.; Haugeland, J. Computing Machinery and Intelligence; MIT Press: Cambridge, MA, USA, 1950. 
24. Turing, A.M. On computable numbers, with an application to the Entscheidungsproblem. Proc. Lond. Math. Soc. 1937, 2, 230-265. [CrossRef]

25. Pfeifer, R.; Scheier, C. Understanding Intelligence; MIT Press: Cambridge, MA, USA, 2001.

26. Sternberg, R.J. Intelligence; John Wiley \& Sons, Inc.: Hoboken, NJ, USA, 2013.

27. Haier, R.J. The Neuroscience of Intelligence; Cambridge University Press: Cambridge, UK, 2016.

28. Blum, M.; Blum, L. A theoretical computer science perspective on consciousness. J. Artif. Intell. Conscious. 2021, 8, 1-42. [CrossRef]

29. Samuel, A.L. Some studies in machine learning using the game of checkers. IBM J. Res. Dev. 1959, 3, 210-229. [CrossRef]

30. Hebb, D.O. The Organisation of Behaviour: A Neuropsychological Theory; Science Editions: New York, NY, USA, 1949.

31. Cybenko, G. Approximation by superpositions of a sigmoidal function. Math. Control Signals Syst. 1989, 2, 303-314. [CrossRef]

32. Available online: https://pytorch.org/ (accessed on 15 April 2021).

33. Available online: https:/ / www.tensorflow.org (accessed on 15 April 2021).

34. Riesenhuber, M.; Poggio, T. Hierarchical models of object recognition in cortex. Nat. Neurosci. 1999, 2, 1019-1025. [CrossRef]

35. Bengio, Y.; Lecun, Y.; Hinton, G. Deep learning for AI. Commun. ACM 2021, 64, 58-65. [CrossRef]

36. Fukunaga, K. Introduction to Statistical Pattern Recognition; Elsevier: Amsterdam, The Netherlands, 2013.

37. James, G.; Witten, D.; Hastie, T.; Tibshirani, R. An Introduction to Statistical Learning; Springer: New York, NY, USA, 2013; Volume 112.

38. Goodfellow, I.; Pouget-Abadie, J.; Mirza, M.; Xu, B.; Warde-Farley, D.; Ozair, S.; Courville, A.; Bengio, Y. Generative adversarial nets. In Advances in Neural Information Processing Systems; Morgan Kaufmann Publishers Inc.: Burlington, MA, USA, 2014; Volume 27.

39. Nguyen, H.; La, H. Review of deep reinforcement learning for robot manipulation. In Proceedings of the 2019 Third IEEE International Conference on Robotic Computing (IRC), Naples, Italy, 25-27 February 2019; pp. 590-595.

40. Nian, R.; Liu, J.; Huang, B. A review on reinforcement learning: Introduction and applications in industrial process control. Comput. Chem. Eng. 2020, 139, 106886. [CrossRef]

41. Levine, S.; Kumar, A.; Tucker, G.; Fu, J. Offline reinforcement learning: Tutorial, review, and perspectives on open problems. arXiv 2020, arXiv:2005.01643.

42. Taylor, L.; Nitschke, G. Improving deep learning with generic data augmentation. In Proceedings of the 2018 IEEE Symposium Series on Computational Intelligence (SSCI), Bangalore, India, 18-21 November 2018; pp. 1542-1547.

43. Wen, Q.; Sun, L.; Yang, F.; Song, X.; Gao, J.; Wang, X.; Xu, H. Time series data augmentation for deep learning: A survey. arXiv 2020, arXiv:2002.12478.

44. Interpretable AI: The Zing-Thing to Bolster the Adoption of AI in Industries. Available online: https://www.linkedin.com/ pulse/interpretable-ai-zing-thing-bolster-adaptability-mishra-phd/ (accessed on 6 August 2021).

45. AI vs. Human: A Comparison of Human Perception with Artificial Intelligence (AI). Available online: https://thinkml.ai/ai-vshuman-comparison-of-human-perception-with-artificial-intelligence/ (accessed on 6 August 2021).

46. Perception Centric AI: Project Rear-View Mirror. Available online: https://amit-india.medium.com/perception-centric-aiproject-rear-view-mirror-df0627aa2030 (accessed on 6 August 2021).

47. Mishra, A.K. ICABiDAS: Intuition centred architecture for big data analysis and synthesis. arXiv 2017, arXiv:1706.00638.

48. Son, J.; Mishra, A.K. A survey of brain inspired technologies for engineering. In Proceedings of the 2016 Pattern Recognition Association of South Africa and Robotics and Mechatronics International Conference (PRASA-RobMech), Stellenbosch, South Africa, 30 November-2 December 2016; pp. 1-6.

49. Kalashnikov, A.; Pakhomovsky, Y.A.; Bazai, A.; Mikhailova, J.; Konopleva, N. Rock-chemistry-to-mineral-properties conversion: Machine learning approach. Ore Geol. Rev. 2021, 136, 104292. [CrossRef]

50. Patel, A.K.; Chatterjee, S.; Gorai, A.K. Development of machine vision-based ore classification model using support vector machine (SVM) algorithm. Arab. J. Geosci. 2017, 10, 107. [CrossRef]

51. Rajabinasab, B.; Asghari, O. Geometallurgical domaining by cluster analysis: Iron ore deposit case study. Nat. Resour. Res. 2019, 28, 665-684. [CrossRef]

52. Lishchuk, V.; Lund, C.; Ghorbani, Y. Evaluation and comparison of different machine-learning methods to integrate sparse process data into a spatial model in geometallurgy. Miner. Eng. 2019, 134, 156-165. [CrossRef]

53. Chauhan, S.; Rühaak, W.; Khan, F.; Enzmann, F.; Mielke, P.; Kersten, M.; Sass, I. Processing of rock core microtomography images: Using seven different machine learning algorithms. Comput. Geosci. 2016, 86, 120-128. [CrossRef]

54. Okada, N.; Maekawa, Y.; Owada, N.; Haga, K.; Shibayama, A.; Kawamura, Y. Automated Identification of Mineral Types and Grain Size Using Hyperspectral Imaging and Deep Learning for Mineral Processing. Minerals 2020, 10, 809. [CrossRef]

55. Deo, A.J.; Sahoo, A.; Behera, S.K.; Das, D.P. Machine Learning based Image Processing for Iron Ore Pellet Size Analysis. In Proceedings of the 2021 th Biennial International Conference on Nascent Technologies in Engineering (ICNTE), Navi Mumbai, India, 15-16 January 2021; pp. 1-5.

56. Tessier, J.; Duchesne, C.; Bartolacci, G. A machine vision approach to on-line estimation of run-of-mine ore composition on conveyor belts. Miner. Eng. 2007, 20, 1129-1144. [CrossRef]

57. Petersen, K.; Aldrich, C.; Van Deventer, J. Analysis of ore particles based on textural pattern recognition. Miner. Eng. 1998, 11, 959-977. [CrossRef] 
58. Tungol, Z.; Kawamura, Y.; Kitahara, I.; Jang, H.D. Development of a Remote Rock Fragmentation Size Distribution Measurement System for Surface Mines Using 3D Photogrammetry. In Proceedings of the 10th International Conference on Explosives and Blasting, Chengdu, China, 27-30 October 2019.

59. Das, S.; Das, D.P.; Sarangi, C.K.; Bhoi, B. Estimation of hydrogen flow rate in atmospheric Ar: $\mathrm{H}_{2}$ plasma by using artificial neural network. Neural Comput. Appl. 2020, 32, 1357-1365. [CrossRef]

60. Nayak, D.K.; Das, D.P.; Behera, S.K.; Das, S.P. Monitoring the fill level of a ball mill using vibration sensing and artificial neural network. Neural Comput. Appl. 2020, 32, 1501-1511. [CrossRef]

61. Van Duijvenbode, J.R.; Buxton, M.W.; Shishvan, M.S. Performance improvements during mineral processing using material fingerprints derived from machine learning-A conceptual framework. Minerals 2020, 10, 366. [CrossRef]

62. Zhou, Y.; Sun, W. Tool wear condition monitoring in milling process based on current sensors. IEEE Access 2020, 8, 95491-95502. [CrossRef]

63. Ostasevicius, V.; Karpavicius, P.; Paulauskaite-Taraseviciene, A.; Jurenas, V.; Mystkowski, A.; Cesnavicius, R.; Kizauskiene, L. A Machine Learning Approach for Wear Monitoring of End Mill by Self-Powering Wireless Sensor Nodes. Sensors 2021, 21, 3137. [CrossRef] [PubMed]

64. Yilmaz, E. Field monitoring and performance evaluation of crushing plant operation. Physicochem. Probl. Miner. Process. 2014, 50, 615-630.

65. Nielsen, K.; Kristiansen, J. Blasting-crushing-grinding: Optimisation of an integrated comminution system. In Rock Fragmentation by Blasting; CRC Press: Boca Raton, FL, USA, 2020; pp. 269-277.

66. Horn, Z.; Auret, L.; McCoy, J.; Aldrich, C.; Herbst, B. Performance of convolutional neural networks for feature extraction in froth flotation sensing. IFAC-PapersOnLine 2017, 50, 13-18. [CrossRef]

67. Pu, Y.; Szmigiel, A.; Chen, J.; Apel, D.B. FlotationNet: A hierarchical deep learning network for froth flotation recovery prediction. Powder Technol. 2020, 375, 317-326. [CrossRef]

68. Pu, Y.; Szmigiel, A.; Apel, D.B. Purities prediction in a manufacturing froth flotation plant: The deep learning techniques. Neural Comput. Appl. 2020, 32, 13639-13649. [CrossRef]

69. Cook, R.; Monyake, K.C.; Hayat, M.B.; Kumar, A.; Alagha, L. Prediction of flotation efficiency of metal sulfides using an original hybrid machine learning model. Eng. Rep. 2020, 2, e12167.

70. Tuşa, L.; Kern, M.; Khodadadzadeh, M.; Blannin, R.; Gloaguen, R.; Gutzmer, J. Evaluating the performance of hyperspectral short-wave infrared sensors for the pre-sorting of complex ores using machine learning methods. Miner. Eng. 2020, 146, 106150. [CrossRef]

71. Cisternas, L.A.; Lucay, F.A.; Botero, Y.L. Trends in modelling, design, and optimization of multiphase systems in minerals processing. Minerals 2020, 10, 22. [CrossRef]

72. Tohry, A.; Jafari, M.; Farahani, M.; Manthouri, M.; Chelgani, S.C. Variable importance assessments of an innovative industrial-scale magnetic separator for processing of iron ore tailings. Miner. Process. Extr. Metall. 2020, 1-8. [CrossRef]

73. Raman, G.S.; Klima, M.S. Application of statistical and machine learning techniques for laboratory-scale pressure filtration: Modeling and analysis of cake moisture. Miner. Process. Extr. Metall. Rev. 2019, 40, 148-155. [CrossRef]

74. Tripathy, S.K.; Murthy, Y.R.; Farrokhpay, S.; Filippov, L.O. Design and analysis of dewatering circuits for a chromite processing plant tailing slurry. Miner. Process. Extr. Metall. Rev. 2021, 42, 102-114. [CrossRef]

75. Ali, D.; Frimpong, S. DeepHaul: A deep learning and reinforcement learning-based smart automation framework for dump trucks. Prog. Artif. Intell. 2021, 10, 157-180. [CrossRef]

76. Olivier, L.E.; Craig, I.K. A survey on the degree of automation in the mineral processing industry. In Proceedings of the 2017 IEEE AFRICON, Cape Town, South Africa, 18-20 September 2017; pp. 404-409.

77. Chai, T.; Ding, J.; Yu, G.; Wang, H. Integrated optimization for the automation systems of mineral processing. IEEE Trans. Autom. Sci. Eng. 2014, 11, 965-982. [CrossRef]

78. Hosseini, S.H.; Samanipour, M. Prediction of final concentrate grade using artificial neural networks from Gol-E-Gohar iron ore plant. Am. J. Min. Metall. 2015, 3, 58-62.

79. Feng, Q.; Zhang, J.; Zhang, X.; Wen, S. Proximate analysis based prediction of gross calorific value of coals: A comparison of support vector machine, alternating conditional expectation and artificial neural network. Fuel Process. Technol. 2015, 129, 120-129. [CrossRef]

80. Wang, J.S.; Han, S. Feed-Forward neural network soft-sensor modelling of flotation process based on particle swarm optimization and gravitational search algorithm. Comput. Intell. Neurosci. 2015, 2015. [CrossRef]

81. Jorjani, E.; Poorali, H.A.; Sam, A.; Chelgani, S.C.; Mesroghli, S.; Shayestehfar, M. Prediction of coal response to froth flotation based on coal analysis using regression and artificial neural network. Miner. Eng. 2009, 22, 970-976. [CrossRef]

82. Jahedsaravani, A.; Marhaban, M.; Massinaei, M. Application of statistical and intelligent techniques for modelling of metallurgical performance of a batch flotation process. Chem. Eng. Commun. 2016, 203, 151-160. [CrossRef]

83. Pan, X.W.; Metzner, G.; Selby, N.; Visser, C.; Letwaba, T.; Coleman, K. Development of weightometer soft sensor. J. S. Afr. Inst. Min. Metall. 2004, 104, 291-296.

84. Ko, Y.D.; Shang, H. A neural network-based soft sensor for particle size distribution using image analysis. Powder Technol. 2011, 212, 359-366. [CrossRef]

85. Ebid, A.M. 35 Years of (AI) in geotechnical engineering: State of the art. Geotech. Geol. Eng. 2021, 39, 637-690. [CrossRef] 
86. Bergen, K.J.; Johnson, P.A.; Maarten, V.; Beroza, G.C. Machine learning for data-driven discovery in solid Earth geoscience. Science 2019, 363. [CrossRef] [PubMed]

87. Global Industry Standard on Tailings Management; Standard, UNEP, ICM and PRI: Nairobi, Kenya, 2020.

88. Morrison, K.F. Preface to the MME Special Focus Issue on Tailings Management. Mining, Metall. Explor. 2021, 38, 1287-1288.

89. Qi, C.; Ly, H.B.; Chen, Q.; Le, T.T.; Le, V.M.; Pham, B.T. Flocculation-dewatering prediction of fine mineral tailings using a hybrid machine learning approach. Chemosphere 2020, 244, 125450. [CrossRef]

90. Nasategay, F.F.U. Detection and Monitoring of Tailings Dam Surface Erosion Using UAV and Machine Learning. Ph.D. Thesis, University of Nevada, Reno, NV, USA, 2020.

91. Betrie, G.D.; Tesfamariam, S.; Morin, K.A.; Sadiq, R. Predicting copper concentrations in acid mine drainage: A comparative analysis of five machine learning techniques. Environ. Monit. Assess. 2013, 185, 4171-4182. [CrossRef] [PubMed]

92. Tousi, E.G.; Duan, J.G.; Gundy, P.M.; Bright, K.R.; Gerba, C.P. Evaluation of E. coli in sediment for assessing irrigation water quality using machine learning. Sci. Total Environ. 2021, 799, 149286. [CrossRef]

93. Hao, L.; Zhang, Z.; Yang, X. Mine tailing extraction indexes and model using remote-sensing images in southeast Hubei Province. Environ. Earth Sci. 2019, 78, 493. [CrossRef]

94. Yan, D.; Li, G.; Li, X.; Zhang, H.; Lei, H.; Lu, K.; Cheng, M.; Zhu, F. An Improved Faster R-CNN Method to Detect Tailings Ponds from High-Resolution Remote Sensing Images. Remote Sens. 2021, 13, 2052. [CrossRef]

95. Rosen, P.A.; Kumar, R. NASA-ISRO SAR (NISAR) Mission Status. In Proceedings of the 2021 IEEE Radar Conference (RadarConf21), Atlanta, GA, USA, 7-14 May 2021; pp. 1-6.

96. Ignatenko, V.; Laurila, P.; Radius, A.; Lamentowski, L.; Antropov, O.; Muff, D. ICEYE Microsatellite SAR Constellation Status Update: Evaluation of first commercial imaging modes. In Proceedings of the IGARSS 2020-2020 IEEE International Geoscience and Remote Sensing Symposium, Waikoloa, HI, USA, 26 September-2 October 2020; pp. 3581-3584.

97. Colliander, A.; Reichle, R.; Crow, W.; Cosh, M.; Chen, F.; Chan, S.; Das, N.; Bindlish, R.; Chaubell, J.; Kim, S.; et al. Validation of Soil Moisture Data Products from the NASA SMAP Mission. TechRxiv 2021. [CrossRef]

98. Vangi, E.; D’Amico, G.; Francini, S.; Giannetti, F.; Lasserre, B.; Marchetti, M.; Chirici, G. The new hyperspectral satellite PRISMA: Imagery for forest types discrimination. Sensors 2021, 21, 1182. [CrossRef]

99. Qin, P.; Cai, Y.; Wang, X. Small waterbody extraction with improved U-Net using Zhuhai-1 hyperspectral remote sensing images. IEEE Geosci. Remote Sens. Lett. 2021, 1-5. [CrossRef]

100. Xiong, R.; Tang, P. Machine learning using synthetic images for detecting dust emissions on construction sites. Smart Sustain. Built Environ. 2021. [CrossRef]

101. Kavitha, N.; Madhumathy, P. Development of an IOT-Based Atmospheric Fine Dust Monitoring System. In Internet of Things, Smart Computing and Technology: A Roadmap Ahead; Springer: New York, NY, USA, 2020; pp. 263-279.

102. Bozdağ, A.; Dokuz, Y.; Gökçek, Ö.B. Spatial prediction of PM10 concentration using machine learning algorithms in Ankara, Turkey. Environ. Pollut. 2020, 263, 114635. [CrossRef]

103. Jiao, Z.; Hu, P.; Xu, H.; Wang, Q. Machine Learning and Deep Learning in Chemical Health and Safety: A Systematic Review of Techniques and Applications. ACS Chem. Health Saf. 2020, 27, 316-334. [CrossRef]

104. Fan, Y.; Zhu, X.; Sui, H.; Sun, H.; Wang, Z. Design and application of toxic and harmful gas monitoring system in fire fighting. Sensors 2019, 19, 369. [CrossRef] [PubMed]

105. Qiu, S.; Chen, B.; Wang, R.; Zhu, Z.; Wang, Y.; Qiu, X. Atmospheric dispersion prediction and source estimation of hazardous gas using artificial neural network, particle swarm optimization and expectation maximization. Atmos. Environ. 2018, 178, 158-163. [CrossRef]

106. Luco, J.; Ferretti, F. QSAR Based on Multiple Linear Regression and PLS Methods for the Anti-HIV Activity of a Large Group of HEPT Derivativess. J. Chem. Inf. Comput. Sci. 1997, 37, 392-401. [CrossRef]

107. Jalali-Heravi, M.; Parastar, F. Use of artificial neural networks in a QSAR study of anti-HIV activity for a large group of HEPT derivatives. J. Chem. Inf. Comput. Sci. 2000, 40, 147-154. [CrossRef]

108. Mishra, A.K.; Patri, O.P. A structure-motivated hybrid machine for prediction of biological activity of chemical compounds. In Proceedings of the 2010 Annual IEEE India Conference (INDICON), Kolkata, India, 17-19 December 2010; pp. 1-4.

109. Quintanilla, P.; Neethling, S.J.; Brito-Parada, P.R. Modelling for froth flotation control: A review. Miner. Eng. 2020, 162, 106718. [CrossRef]

110. von Rueden, L.; Mayer, S.; Sifa, R.; Bauckhage, C.; Garcke, J. Combining machine learning and simulation to a hybrid modelling approach: Current and future directions. In International Symposium on Intelligent Data Analysis; Springer: Cham, Switzerland, 2020; pp. 548-560.

111. Blei, D.M.; Ng, A.Y.; Jordan, M.I. Latent dirichlet allocation. J. Mach. Learn. Res. 2003, 3, 993-1022.

112. Moser, G.; Lee, S.H.; Hayes, B.J.; Goddard, M.E.; Wray, N.R.; Visscher, P.M. Simultaneous discovery, estimation and prediction analysis of complex traits using a Bayesian mixture model. PLoS Genet. 2015, 11, e1004969. [CrossRef]

113. Ren, J.; Ni, D. A batch-wise LSTM-encoder decoder network for batch process monitoring. Chem. Eng. Res. Des. 2020, 164, 102-112. [CrossRef]

114. Liu, K.; Zhang, J. A Dual-Layer Attention-Based LSTM Network for Fed-batch Fermentation Process Modelling. In Computer Aided Chemical Engineering; Elsevier: Amsterdam, The Netherlands, 2021; Volume 50, pp. 541-547.

115. Halle, M.; Stevens, K. Speech recognition: A model and a program for research. IRE Trans. Inf. Theory 1962, 8, 155-159. [CrossRef] 
116. Reddy, D.R. Speech recognition by machine: A review. Proc. IEEE 1976, 64, 501-531. [CrossRef]

117. Juang, B.H.; Rabiner, L.R. Automatic Speech Recognition-A Brief History of the Technology Development; Georgia Institute of Technology; Atlanta Rutgers University and the University of California: Santa Barbara, CA, USA, 2005; p. 67.

118. Jaitly, N.; Nguyen, P.; Senior, A.; Vanhoucke, V. Application of Pretrained Deep Neural Networks to Large Vocabulary Speech Recognition. In Proceedings of the Interspeech 2012, Portland, OR, USA, 9-13 September 2012.

119. Eirinakis, P.; Kalaboukas, K.; Lounis, S.; Mourtos, I.; Rožanec, J.M.; Stojanovic, N.; Zois, G. Enhancing cognition for digital twins. In Proceedings of the 2020 IEEE International Conference on Engineering, Technology and Innovation (ICE/ITMC), Cardiff, UK, 15-17 June 2020; pp. 1-7.

120. Zobel-Roos, S.; Schmidt, A.; Uhlenbrock, L.; Ditz, R.; Köster, D.; Strube, J. Digital Twins in Biomanufacturing. In Digital Twins; Springer: New York, NY, USA, 2020; pp. 181-262.

121. Schilling, D.R. Knowledge doubling every 12 months, soon to be every 12 hours. Industry Tap, 19 April 2013.

122. Brown, T. Design thinking. Harv. Bus. Rev. 2008, 86, 84 .

123. Gómez, D.; Rojas, A. An empirical overview of the no free lunch theorem and its effect on real-world machine learning classification. Neural Comput. 2016, 28, 216-228. [CrossRef] [PubMed]

124. Adam, S.P.; Alexandropoulos, S.A.N.; Pardalos, P.M.; Vrahatis, M.N. No free lunch theorem: A review. Approx. Optim. 2019, 145, 57-82.

125. Marcum, J. A statistical theory of target detection by pulsed radar. IRE Trans. Inf. Theory 1960, 6, 59-267. [CrossRef]

126. Rengasamy, D.; Jafari, M.; Rothwell, B.; Chen, X.; Figueredo, G.P. Deep learning with dynamically weighted loss function for sensor-based prognostics and health management. Sensors 2020, 20, 723. [CrossRef]

127. Beygelzimer, A.; Dasgupta, S.; Langford, J. Importance weighted active learning. In Proceedings of the 26th Annual International Conference on Machine Learning, Montreal, QC, Canada, 14-18 June 2009; pp. 49-56.

128. Zhang, W.; Du, Y.; Yoshida, T.; Yang, Y. DeepRec: A deep neural network approach to recommendation with item embedding and weighted loss function. Inf. Sci. 2019, 470, 121-140. [CrossRef]

129. Czech, D.; Mishra, A.; Inggs, M. A CNN and LSTM-based approach to classifying transient radio frequency interference. Astron. Comput. 2018, 25, 52-57. [CrossRef]

130. Bezerra, A.; Silva, I.; Guedes, L.A.; Silva, D.; Leitão, G.; Saito, K. Extracting value from industrial alarms and events: A data-driven approach based on exploratory data analysis. Sensors 2019, 19, 2772. [CrossRef]

131. Taboada, G.L.; Han, L. Exploratory data analysis and data envelopment analysis of urban rail transit. Electronics 2020, 9, 1270. [CrossRef]

132. Czech, D.; Mishra, A.; Inggs, M. Characterizing transient radio-frequency interference. Radio Sci. 2017, 52, 841-851. [CrossRef]

133. Jolliffe, I.T.; Cadima, J. Principal component analysis: A review and recent developments. Philos. Trans. R. Soc. A Math. Phys. Eng. Sci. 2016, 374, 20150202. [CrossRef]

134. Czech, D.; Mishra, A.K.; Inggs, M. A canonical interferencelet-based approach to RFI identification. In Proceedings of the 2016 Radio Frequency Interference (RFI), Socorro, NM, USA, 17-20 October 2016; pp. 16-20.

135. Loh, P.L. On lower bounds for statistical learning theory. Entropy 2017, 19, 617. [CrossRef]

136. Piou, J.E. Computation of Posterior Cramer-Rao Bounds for Deep Learning Networks. In Proceedings of the 2020 11th IEEE Annual Ubiquitous Computing, Electronics \& Mobile Communication Conference (UEMCON), New York, NY, USA, 28-31 October 2020; pp. 0240-0244.

137. Hannun, A.; Guo, C.; van der Maaten, L. Measuring Data Leakage in Machine-Learning Models with Fisher Information. arXiv 2021, arXiv:2102.11673.

138. Petroff, E.; Keane, E.; Barr, E.; Reynolds, J.; Sarkissian, J.; Edwards, P.; Stevens, J.; Brem, C.; Jameson, A.; Burke-Spolaor, S.; et al. Identifying the source of perytons at the Parkes radio telescope. Mon. Not. R. Astron. Soc. 2015, 451, 3933-3940. [CrossRef]

139. Schneider, S.; Murphy, R.J.; Melkumyan, A. Evaluating the performance of a new classifier-the GP-OAD: A comparison with existing methods for classifying rock type and mineralogy from hyperspectral imagery. ISPRS J. Photogramm. Remote Sens. 2014, 98, 145-156. [CrossRef]

140. Morkun, V.; Tron, V.; Goncharov, S. Automation of the ore varieties recognition process in the technological process streams based on the dynamic effects of highenergy ultrasound. Metall. Min. Ind. 2015, 2, 31-34.

141. Gewali, U.B.; Monteiro, S.T.; Saber, E. Machine learning based hyperspectral image analysis: A survey. arXiv 2018, arXiv:1802.08701.

142. Jiang, Y.; Yin, S.; Dong, J.; Kaynak, O. A review on soft sensors for monitoring, control and optimization of industrial processes. IEEE Sens. J. 2021, 21, 12868-12881. [CrossRef]

143. Sardar, S.; Mishra, A.K. ASIN-based UWB radar for sludge monitoring. IEEE Access 2014, 2, 290-300. [CrossRef]

144. Mishra, A.K. Application Specific Instrumentation (ASIN): A Bio-inspired Paradigm to Instrumentation using recognition before detection. arXiv 2016, arXiv:1611.00228. 